\title{
The Development of a Syntactic Awareness Task using the Word-Order Correction Paradigm
}

\section{Background}

- Syntactic awareness refers to the ability to reflect on the sentence structure (Cain, 2007), and it is found to predict reading comprehension in children (e.g Mokhtari \& Thompson, 2006; Muter et al., 2004; Nation et al., 2010).

- This poster described the development of a Syntactic Awareness task that stemmed from a larger project exploring the possible interplay of the psycholinguistic and cognitive predictors of reading comprehension in British and Chinese children. The project measured children's Syntactic Awareness, along with Reading Comprehension, Vocabulary, Discourse Coherence, Executive Functions (Cognitive Flexibility, Inhibition Planning, Working Memory), Metamemory, Short-term Memory, and Nonverbal Reasoning.

\section{Research Questions}

- To develop an appropriate measure of Syntactic Awareness in English

- To examine the psychometric properties of this experimentally designed Syntactic Awareness instrument

\section{Methods}

- A word-order correction paradigm, ie. rearranging the fragmented words or phrases into logical and meaningful sentences (Brimo et al., 2017; Cain, 2007; Nation \& Snowling, 2000), was adopted in the development of the measurement.

- Based on Nation and Snowling's (2000) findings, items were developed with varying degree of syntactic complexity (the grammatical structure of the sentences) and semantic ambiguity (the possible correct combinations of the words) by adapting the materials from their study.

- Compared to Nation and Snowling's (2000) materials, items on this task demonstrated more variations in their grammatical structures.

- As the items on the instrument were designed to accommodate different sentence combinations, the responses were coded and scored using the following criteria:

$\square$ Preferred (2): both syntactically correct and semantically possible (i.e. it makes sense)

$\square$ Non-preferred (1): syntactically correct but semantically impossible (i.e. it does not make sense)

$\square$ Incorrect (0): syntactically incorrect

Example: (1) the banana / (2) the mouse / (3) pushing / (4) that / (5) was / (6) ate / (7) the man

Answer \#1: (7) the man - (5) was - (3) pushing - (2) the mouse - (4) that - (6) ate - (1) the banana [Preferred] Answer \#2: (1) the banana - (4) that - (5) was - (3) pushing - (2) the mouse - (6) ate - (7) the man [Not-preferred] Answer \#3: (1) the banana - (2) the mouse - (3) pushing - (4) that - (5) was - (6) ate - (7) the man [Incorrect]

- The experimentally designed Syntactic Awareness instrument was first piloted on 16 Year 5 children $(M=10.37)$ and 16 Year 9 children $(M=13.49)$ respectively. Changes were made based on the pilot study and the final version consisted of six items.

- The instrument was then administered to 325 Year 5 and Year 6 children, of which 242 also completed other measures from the aforementioned larger project.

\section{Results}

$(\mathrm{N}=325)$

- Inter-rater reliability: $96.14 \%$

- Cronbach's Alpha: 0.69

- Split-half reliability: 0.70

- Composite reliability: 0.70

- Exploratory factor analysis confirmed a one-factor model

$(\mathrm{N}=242)$

- Kendall's Tau indicated that Syntactic Awareness was positively correlated with Reading Comprehension $(\mathrm{Tb}=.27, \mathrm{p}<.01)$ Vocabulary $(\mathrm{Tb}=.33, \mathrm{p}<.01)$ Discourse Coherence $(\mathrm{Tb}=.36, \mathrm{p}<.01)$, Planning $(\mathrm{Tb}=.15, \mathrm{p}<.05)$ Working Memory $(\mathrm{Tb}=.22, \mathrm{p}<.01)$ Short-term Memory $(\mathrm{Tb}=.27, \mathrm{p}<.01)$ Non-verbal Reasoning $(\mathrm{Tb}=.15, \mathrm{p}<.05)$

\section{Conclusion}

- The findings indicated the suitability of this six-item instrument as a measure of Syntactic Awareness.

- Future studies that aim to examine Syntactic Awareness on its own or in relation to reading ability could use this task as the measurement of the construct.

- The extent to which this instrument could be applied in different linguistic setting should be investigated. 